\title{
Age-Adjusted PSA
}

National Cancer Institute

\section{Source}

National Cancer Institute. Age-Adjusted PSA. NCI Thesaurus. Code C20120.

Prostate-specific antigen (PSA) level defined according to specific age groups or ranges,

which takes into account the natural increase of PSA levels with increased age. 